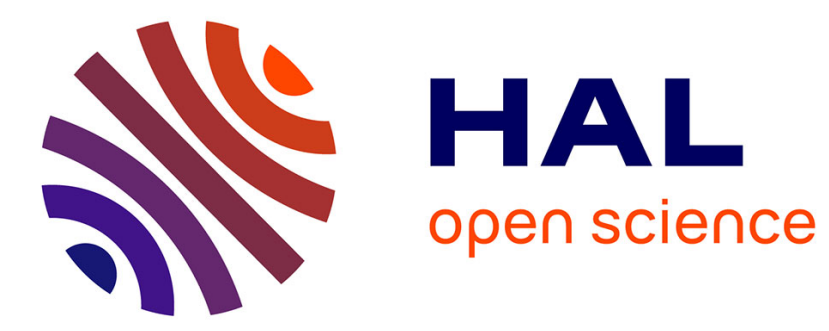

\title{
Microtearing turbulence: magnetic braiding and disruption limit
}

\author{
Marie-Christine Firpo
}

\section{To cite this version:}

Marie-Christine Firpo. Microtearing turbulence: magnetic braiding and disruption limit. Physics of Plasmas, 2015, 22, pp.122511 10.1063/1.4938273 . hal-01248802

\section{HAL Id: hal-01248802 \\ https://hal.science/hal-01248802}

Submitted on 28 Dec 2015

HAL is a multi-disciplinary open access archive for the deposit and dissemination of scientific research documents, whether they are published or not. The documents may come from teaching and research institutions in France or abroad, or from public or private research centers.
L'archive ouverte pluridisciplinaire HAL, est destinée au dépôt et à la diffusion de documents scientifiques de niveau recherche, publiés ou non, émanant des établissements d'enseignement et de recherche français ou étrangers, des laboratoires publics ou privés. 


\title{
Microtearing turbulence: magnetic braiding and disruption limit
}

\author{
Marie-Christine Firpo ${ }^{1}$ \\ Laboratoire de Physique des Plasmas, CNRS - Ecole Polytechnique, 91128 Palaiseau cedex, \\ France
}

A realistic reduced model involving a large poloidal spectrum of microtearing modes is used to probe the existence of some stochasticity of magnetic field lines. Stochasticity is shown to occur even for the low values of the magnetic perturbation $\delta B / B$ devoted to magnetic turbulence that have been experimentally measured. Because the diffusion coefficient may strongly depend on the radial (or magnetic-flux) coordinate, being very low near some resonant surfaces, and because its evaluation implicitly makes a normal diffusion hypothesis, one turns to another indicator appropriate to diagnose the confinement: the mean residence time of magnetic field lines. Their computation in the microturbulence frame points to the existence of a disruption limit, namely of a critical order of magnitude of $\delta B / B$ above which stochasticity is no longer benign yet leads to a macroscopic loss of confinement in some tens to hundred of electron toroidal excursions. Since the level of magnetic turbulence $\delta B / B$ has been measured to grow with the plasma electron density this would also be a density limit.

\section{INTRODUCTION AND OBJECTIVES}

In the tokamak terminology, magnetic microturbulence refers to the simultaneous excitation of a large spectrum of magnetic modes with poloidal mode numbers $m \gg 1$. It might however be thought that the smallness of the relative magnitude of the magnetic perturbation $\delta B / B$ devoted to magnetic turbulence as measured in some tokamak experiments ${ }^{1,2}$ makes the question of the stochasticity of the magnetic field lines rather irrelevant. Yet, several recent results coming from gyrokinetic simulations have unveiled the ubiquity of the magnetic stochasticity under physical parameters relevant to fusion-oriented tokamak plasmas. In particular, electromagnetic numerical simulations in the conditions of tokamak ion temperature gradient turbulence ${ }^{3}$ have demonstrated that the magnetic field could be stochastic even at very low plasma pressure. Concurrently, another study ${ }^{8}$ aiming at unveiling the nature and mechanisms behind turbulent transport in tokamak plasmas based on $a b$ initio gyrokinetic simulations demonstrated that heat transport is dominated by the electron magnetic component and pointed to microtearing modes as a possible candidate to explain turbulent transport in tokamak plasmas. Moreover, other nonlinear gyrokinetic numerical simulations of microtearing mode turbulence using the experimental parameters from a high- $\beta$ discharge of the spherical torus NSTX experiment ${ }^{9}$ indicated that the transport is almost entirely electromagnetic as a result of the electrons diffusing in the stochastic magnetic field. Lastly, electromagnetic gyrokinetic simulations of plasma microturbulence ${ }^{10}$ have also supported the nearubiquitous character of magnetic stochasticity.

Experimentally, there is increasing evidence of the microtearing nature of magnetic turbulence. Very recently, the first direct experimental verification of microtearing mode turbulence in the core region of tokamak plasmas has been reported in the JIPPT-IIU tokamak ${ }^{2}$, where local magnetic fluctuations were measured using a heavy ion beam probe. In the ASDEX-Upgrade, edge measurements using electron cyclotron emission imaging just detected also that the temperature fluctuations have features similar to microtearing modes ${ }^{11}$. Accordingly, there is now a growing body of evidence both from numerical gyrokinetic simulations and novel experimental diagnostic approaches that magnetic fluctuations come under the form of microtearing modes that control electron heat transport.

Gyrokinetic numerical simulations are certainly useful since they should eventually provide a fine-scale selfconsistent picture of tokamak plasma dynamics, yet they are highly numerically-demanding and challenging. It remains therefore desirable to continue to build and develop reduced and versatile models to improve the understanding of the puzzling and critical tokamak transport issue and to test paths towards the improvement of the confinement. In order to reduce the complexity of the system formed by the strong equilibrium magnetic field and the assemblies of charged particles selfconsistently interacting with turbulent electromagnetic perturbations, one may adopt a non self-consistent test particle approach under suitable assumptions for the electromagnetic field. A more fundamental approach is to focus on the magnetic structure of the confinement. Indeed the Maxwell equation $\operatorname{div} \mathbf{B}=0$ forms a closed and exact equation enabling to isolate the effect of the magnetic field and study its properties. This does not mean at all that one neglects the electric field or assumes the nullity of the electric perturbations. This does not mean either that the trajectories of charged particles in a spatially integrable magnetic field are integrable since the threedegrees of freedom Hamiltonian from which derive the particle equations of motion may a priori allow chaotic motions. Indeed in the case where the (integrable) magnetic structure possesses an X-point, as in the divertor scheme, charged particles experience a chaotic motion in the vicinity of the magnetic separatrices ${ }^{12}$. This is just a reduction of the picture. It was notably shown by Cary and Littlejohn in a seminal paper ${ }^{13}$ that the zero divergence of the magnetic field, which may be interpreted as a condition of phase space conservation, makes the equations of magnetic field lines derive from a Hamiltonian 
system.

This line of research connected years ago to the introduction of the paradigmatic standard map ${ }^{14}$ that had a considerable importance in the understanding of the onset and phase-space manifestations of Hamiltonian chaos. This captured the universal behaviour of area-preserving maps with divided phase space when integrable islands of stability are surrounded by a chaotic component. Yet, this model is not meant to address the quantitative transport issues posed by magnetic confinement fusion. Using the present computing capacities and the improved knowledge of the magnetic turbulence coming from experimental measurements and recent gyrokinetic results, it has become possible to consider more realistic models of magnetic field lines under microturbulence conditions and compute their transport properties. The microturbulence magnetic framework used in the present study will be exposed in Section II. Some numerical results obtained within such microtearing turbulence models having a large spectrum of poloidal modes will be presented. It will be shown that a value of the magnitude of the magnetic perturbation as low as $10^{-5}$ does not imply that magnetic field lines are close to integrability.

The problem of the disruptions in toroidal devices for magnetic confinement fusion must be tamed to ensure the viability of industrial fusion reactors. Contrarily to macroscopic dramatic phenomena, such as the sawtooth crash, that may be related to the onset of the chaos of magnetic field lines due to some subset of longwavelength modes with different helicities (See e.g. ${ }^{4-7}$ ), the consequences of the loss of the integrity of magnetic surfaces in the presence of plasma microturbulence have been observed in some gyrokinetic numerical simulations $^{3}$ to be normally benign or moderate. Yet, there is certainly some limit in the braiding of the magnetic field lines above which magnetic confinement breaks and a disruption occurs. Section III will be devoted to the exploration of disruption limits associated to magnetic microturbulence. This study will involve the evaluation of some probability distribution functions (pdf) of magnetic confinement times. These are obtained through rather demanding numerical simulations within the reduced magnetic turbulence model. Yet, it is useful to point out the benefit of using a reduced approach by noting that the numerical cost of analogous simulations within a full gyrokinetic frame would have been much higher and possibly prohibitive. A short discussion concludes the work.

\section{THE HAMILTONIAN MICROTEARING TURBULENCE FRAMEWORK}

\section{A. Presentation}

The canonical representation of an arbitrary magnetic field in a toroidal magnetic confinement device is written in Eq. (1). Let us consider a set of variables $(\rho, \theta, \phi)$ where $\rho$ is a radius-like variable that vanishes along the magnetic axis. Then, there exist ${ }^{15}$ two single-valued functions $\psi(\rho, \theta, \phi)$ and $\Phi(\rho, \theta, \phi)$, such that the magnetic field can be written in the form

$$
\mathbf{B}=\nabla \psi \times \nabla \theta+\nabla \phi \times \nabla \Phi .
$$

Eliminating the variable $\rho$ by using $\Phi(\psi, \theta, \phi)$, the equations for the magnetic field lines read

$$
\begin{aligned}
\frac{d \psi}{d \phi} & =\frac{\mathbf{B} \cdot \nabla \psi}{\mathbf{B} \cdot \nabla \phi}=-\frac{\partial \Phi}{\partial \theta}, \\
\frac{d \theta}{d \phi} & =\frac{\mathbf{B} \cdot \nabla \theta}{\mathbf{B} \cdot \nabla \phi}=\frac{\partial \Phi}{\partial \psi} .
\end{aligned}
$$

In this representation, $\Phi$ plays the role of a generically one-and-a-half degrees of freedom Hamiltonian ${ }^{16-18}$, the toroidal angle $\phi$ is a time-like variable and $\psi$ is the momentum conjugated to the poloidal angle $\theta$. In a nonsteady state, the field-line Hamiltonian depends also on the real time $t$, that plays the role of a parameter, but not of a canonical variable, and will be noted $\Phi^{t}$.

In tokamaks, the equilibrium configuration is axisymmetric. The equilibrium magnetic field $\mathbf{B}_{0}(\mathbf{r})$ has its field lines spiralling on perfect nested magnetic surfaces around the magnetic axis and these are defined by a constant $\psi$. This amounts to say that the magnetic fieldline Hamiltonian associated to $\mathbf{B}_{0}$ is integrable, being $\Phi_{0}(\psi)$. It identifies with the poloidal magnetic flux and $\psi$ with the toroidal magnetic flux. From the equilibrium poloidal flux $\Phi_{0}(\psi)$, one defines the very important quantity called the safety factor profile $q(\psi)$, or equivalently its inverse, the winding profile $w(\psi)$, through

$$
\frac{d \Phi_{0}(\psi)}{d \psi}=\frac{1}{q(\psi)}=w(\psi) .
$$

Yet, axisymmetry is bound to be broken by external effects, such as the toroidal ripple in the magnetic field, which appears in tokamaks due to the finite number of toroidal magnetic field coils, or as the edge magnetic perturbations due to additional coils serving to control particle and heat loads on tokamak walls ${ }^{19}$ or by some intrinsic instability phenomena such as MHD activity or microturbulence. The generic form of the magnetic fieldline Hamiltonian reads then

$$
\Phi^{t}(\psi, \theta, \phi)=\Phi_{0}(\psi)+\delta \Phi^{t}(\psi, \theta, \phi) .
$$

The order of magnitude of the relative perturbation $|\delta \mathbf{B}| / B_{0}$ quantifies the deviation from axisymmetry and integrability. Using the poloidal and toroidal periodicities, this can be Fourier decomposed as

$$
\delta \Phi(\psi, \theta, \phi)=\sum_{m, n} \varepsilon_{m, n} \delta \Phi_{m n}(\psi) \cos \left(m \theta-n \phi+\chi_{m n}\right),
$$

where from now on, the time dependence index has been dropped to simplify the notations. A rough model for the behavior of microtearing waveforms was proposed by 
Stix in $^{28}$. In the present work, a more refined model of microtearing turbulence described in $^{27}$ will be used. This is an empirical experimentally-based model taking

$$
\delta \Phi_{m n}(\psi) \equiv\left[\cosh \left(\frac{1}{\Delta} \ln \frac{\psi}{\psi_{m n}}\right)\right]^{-m \frac{\Delta}{2}} .
$$

The $(m, n)$ waveform is maximal on the rational surface $\psi_{m n}$, defined as the value of the toroidal magnetic flux for which $q\left(\psi_{m n}\right)=m / n$. The physically realistic value of the parameter $\Delta$ is about 0.01 . This ensures the correct minimally-smooth peaking of the waveforms. In numerical simulations, the poloidal modes with $1<m<40$ have been retained and the associated toroidal modes are such that the resonances $(m, n)$ are present. Figure 1 shows these modes. A standard winding profile is used with

$$
w(\psi)=0.2(2-\psi)\left(\psi^{2}-2 \psi+2\right),
$$

corresponding to a monotonously growing $q$-profile. It

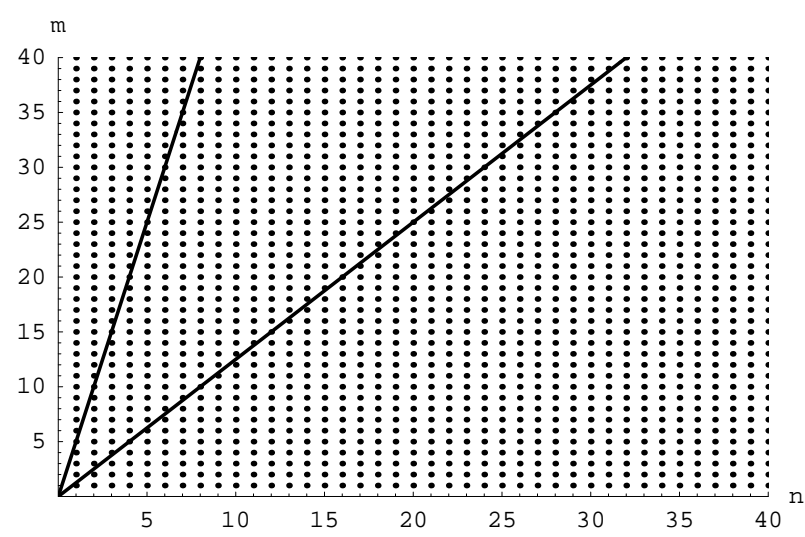

FIG. 1. The $(m, n)$ modes retained in this study are within the limits imposed by the minimal and maximal $q$-values defining respectively the lower and upper lines.

should be noted that the present model considers the whole spectrum of magnetic modes, not the only the part with the largest $m$ modes that may be properly defined as the turbulent part. This is consistent with the experimental reality where relatively large scale magnetohydrodynamic-like modes coexist with small scale perturbations.

\section{B. The Hamiltonian for a single mode: estimation of the resonance widths}

Let us first characterize the Hamiltonian model in the case of a single arbitrary mode $\left(m_{0}, n_{0}\right)$. Using a canonical transform with the generating function $F_{2}(\theta, \Psi, \phi)=\left(m_{0} \theta-n_{0} \phi\right) \Psi$, that amounts to moving to the $\left(m_{0}, n_{0}\right)$ wave frame, the single mode Hamiltonian reads $\widehat{\Phi}(\Psi, \Theta, \phi)=\Phi(\psi, \theta, \phi)+\partial F_{2} / \partial \phi$ with $\psi=m_{0} \Psi$ and $\Theta=m_{0} \theta-n_{0} \phi$. This yields

$\widehat{\Phi}(\Psi, \Theta)=\Phi_{0}\left(m_{0} \Psi\right)-n_{0} \Psi+\varepsilon_{m_{0}, n_{0}} \delta \Phi_{m_{0} n_{0}}\left(m_{0} \Psi\right) \cos \Theta$.

Figure 2 and Figure 3 represent respectively the perturbed Hamiltonian waveform and the topology of the Hamiltonian phase space for the same one-wave integrable case. The fixed points of the Hamiltonian (9)

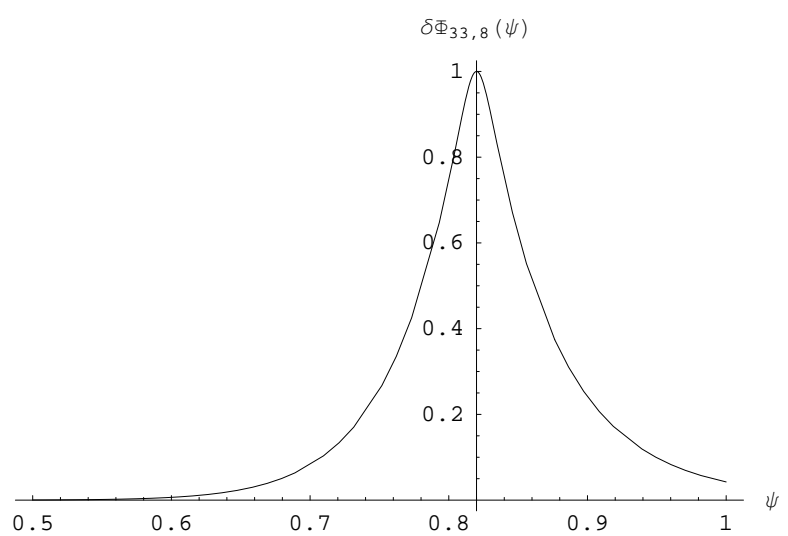

FIG. 2. Perturbed Hamiltonian waveform $\delta \Phi_{m_{0} n_{0}}$ for the mode $m_{0}=33, n_{0}=8$ and for the parameter $\Delta=0.01$. The $q$-profile considered here is the same as in Figure 3.

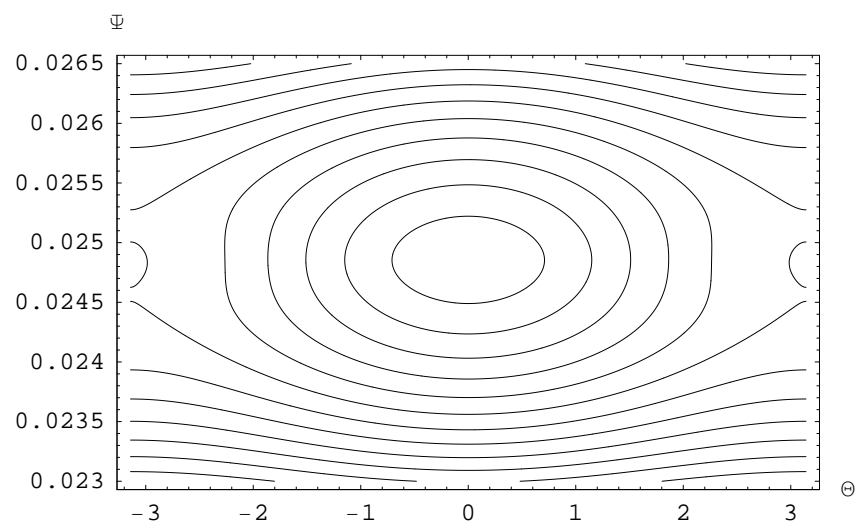

FIG. 3. Phase space portrait of magnetic field lines in the case where a single microtearing mode is retained, displayed in the wave frame in the $(\Theta, \Psi)$ space. Here this is the mode $m_{0}=33, n_{0}=8$. The $q$-profile used in the figure is $q(\psi)=$ $1.5+3.2 \psi$ which gives $\psi_{m_{0}=33, n_{0}=8} \simeq 0.82$ that corresponds, in the new wave frame variables, to $\Psi \simeq 0.248$. The amplitude of the mode is $\varepsilon_{m_{0}, n_{0}}=5.10^{-4}$.

are given by $\Theta=0$ or $\pi$. For $\Theta=\pi$, the action $\psi$ of the fixed points satisfies

$0=\frac{1}{q(\psi)}-\frac{n_{0}}{m_{0}}+\varepsilon_{m_{0}, n_{0}} \frac{m_{0}}{2 \psi} \delta \Phi_{m_{0} n_{0}}(\psi) \tanh \left(\frac{1}{\Delta} \ln \frac{\psi}{\psi_{m_{0} n_{0}}}\right)$.

This equations admits an obvious solution $\psi=\psi_{m_{0} n_{0}}$ corresponding to an elliptic fixed point and it admits two other roots $\psi^{+}>\psi_{m_{0} n_{0}}$ and $\psi^{-}<\psi_{m_{0} n_{0}}$ in the 
case where the $q$-profile is monotonously increasing that correspond to hyperbolic fixed points. We can then estimate the resonance width associated to the $\left(m_{0}, n_{0}\right)$ single wave Hamiltonian. The largest toroidal flux corresponding to the upper half-width of the resonance, denoted by $\bar{\psi}^{+}$, may be estimated from the energy conservation on the upper separatrix so that $\widehat{\Phi}\left(\psi^{+} / m_{0}, \pi\right)=$ $\widehat{\Phi}\left(\bar{\psi}^{+} / m_{0}, 0\right)$. Under the assumption that $\bar{\psi}^{+} \simeq \psi^{+}$, this yields

$$
\bar{\psi}^{+}-\psi^{+} \sim \varepsilon_{m_{0}, n_{0}} \frac{\delta \Phi_{m_{0} n_{0}}\left(\bar{\psi}^{+}\right)+\delta \Phi_{m_{0} n_{0}}\left(\psi^{+}\right)}{\frac{n_{0}}{m_{0}}-\frac{1}{q\left(\bar{\psi}^{+}\right)}} .
$$

Finally, for the consistent hypotheses ensuring the smallness of $\bar{\psi}^{+}-\psi^{+}$, namely $\varepsilon_{m_{0}, n_{0}}$ sufficiently small and a non-vanishing magnetic shear at the rational surface, it is possible to further simplify Eq. (11) by making $\psi^{+} \simeq$ $\psi_{m_{0} n_{0}}, \delta \Phi_{m_{0} n_{0}}\left(\bar{\psi}^{+}\right) \simeq \delta \Phi_{m_{0} n_{0}}\left(\psi^{+}\right) \simeq 1$, and by using $q\left(\bar{\psi}^{+}\right)-m_{0} / n_{0} \simeq q^{\prime}\left(m_{0} / n_{0}\right)\left(\psi^{+}-\psi_{m_{0} n_{0}}\right)$. The expression of the resonance half-width, $\delta \psi_{m_{0} n_{0}}=\bar{\psi}^{+}-\psi_{m_{0} n_{0}}$, amounts then to

$$
\delta \psi_{m_{0} n_{0}}=\left(\frac{2 \varepsilon_{m_{0}, n_{0}}}{\left|w^{\prime}\left(\psi_{m_{0} n_{0}}\right)\right|}\right)^{1 / 2}=\frac{m_{0}}{n_{0}}\left(\frac{2 \varepsilon_{m_{0}, n_{0}}}{q^{\prime}\left(\psi_{m_{0} n_{0}}\right)}\right)^{1 / 2} .
$$

This is the classical expression of the microtearing resonance half-width.

Unless otherwise specified, we define $\varepsilon$ such that $\varepsilon_{m, n} \equiv \varepsilon n / m$, so that the mode amplitudes $\varepsilon_{m, n}$ are indeed all of the same order $\varepsilon$. The expression of the resonance half-widths reduces then to $\delta \psi_{m_{0} n_{0}}=$ $\left[2 \varepsilon q\left(\psi_{m_{0} n_{0}}\right) / q^{\prime}\left(\psi_{m_{0} n_{0}}\right)\right]^{1 / 2}$. The magnetic field perturbation $\delta B_{m n}$ relates to $\varepsilon_{m, n}$ through

$$
\frac{\delta B_{m n}}{B_{0 \phi}}=\frac{1}{2 \sqrt{2}} \frac{m \varepsilon_{m, n}}{A \sqrt{\psi_{m n}}}
$$

where $A$ denotes the tokamak aspect ratio $\left(A=R_{0} / a\right)$. Although the knowledge of the magnetic spectrum would be necessary to make quantitative estimations, which remains a really challenging task due to the experimental difficulties to measure internal magnetic perturbations, the objective of the present study is more qualitative than quantitative so that we shall mainly retain for our purposes that $\delta B / B \propto \varepsilon$.

\section{Characterization of the local magnetic stochasticity}

The previous expression of the microtearing resonance half-widths may serve to estimate the local Chirikov parameter that quantifies the local chaoticity of the Hamiltonian magnetic field lines. The expression of the Chirikov parameter relative to any couple of modes $(m, n)$ and $\left(m^{\prime}, n^{\prime}\right)$ reads

$$
s_{(m, n),\left(m^{\prime}, n^{\prime}\right)}=\frac{\delta \psi_{m n}+\delta \psi_{m^{\prime} n^{\prime}}}{\left|\psi_{m n}-\psi_{m^{\prime} n^{\prime}}\right|} .
$$

Figure 4 represents the local maximal Chirikov parameter associated to the modes $(m, n)$. More precisely, for each resonant surface $\psi_{m n}$ is plotted the maximal Chirikov parameter $s\left(\psi_{m n}\right)=\max _{\left(m^{\prime}, n^{\prime}\right)} s_{(m, n),\left(m^{\prime}, n^{\prime}\right)}$ divided by $\sqrt{\varepsilon}$ that the mode $(m, n)$ realizes with some other mode $\left(m^{\prime}, n^{\prime}\right)$. The extent of the local maximal stochasticity zone is represented by an horizontal segment between each couple $\left(\psi_{m n}, \psi_{m^{\prime} n^{\prime}}\right)$. From Figure 4 , it is clear that

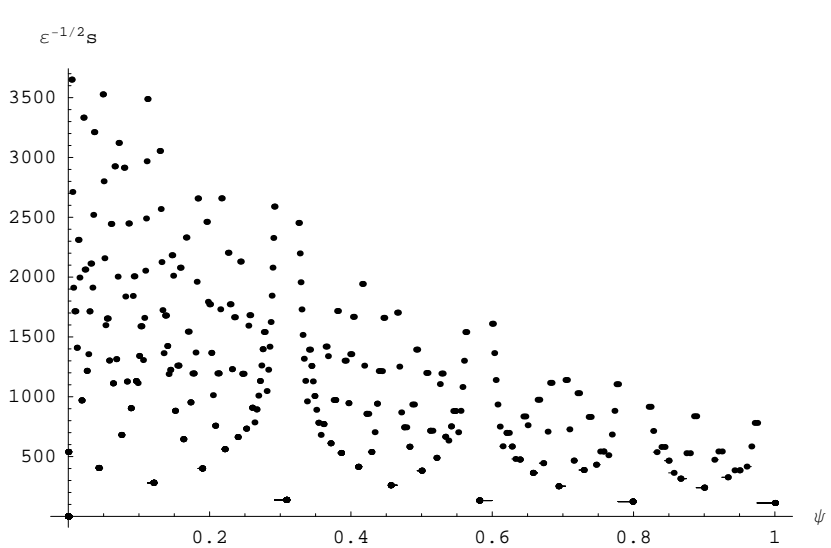

FIG. 4. For each resonant surface $\psi_{m n}$ on the X-axis is plotted by a point the maximal Chirikov parameter $s$ divided by $\sqrt{\varepsilon}$ realized with some mode $\left(m^{\prime}, n^{\prime}\right)$ among the available modes. The segments between the $\psi_{m n}$ and their associated $\psi_{m^{\prime} n^{\prime}}$ are marked but are only wide enough to be visible about the resonances associated to the integer values of $q$. The $q$-profile used in the calculations corresponds to Eq. (8).

there are local minima of stochasticity in the close vicinity of the resonances associated to integer values of the $q$-profile while there are local maxima of stochasticity just in the periphery of these zones. This result is valid for $q$-profiles having a non-vanishing magnetic shear, that is a condition for the validity of Eq. (12), and is consistent with experimental results indicating that transport barriers preferentially form about surfaces where $q$ is an integer.

Another consequence of the results shown on Figure 4 is that, apart from those neighborhoods of the $q=$ integer and low rational surfaces, some mid scale stochasticity is realized even at extremely low values of the relative magnetic perturbation amplitude $\varepsilon$, in our case even at $\varepsilon \sim 10^{-7}-10^{-6}$. Experimentally, $\varepsilon$ has been reported ${ }^{1}$ to be of the order of $10^{-5}$ to $10^{-4}$ (in the $\mathrm{L}$ mode) in Tore Supra and, very recently, to be of order $10^{-4}$ in the JIPPT-IIU tokamak plasmas ${ }^{2}$. Therefore, within the microtearing turbulence frame, the stochasticity of the magnetic field lines under experimentally relevant values of $\varepsilon$ is generic. This is in agreement with the seminal Stix's analysis ${ }^{28}$ and the aforementioned recent gyrokinetic results ${ }^{3,8-10}$. 


\section{Numerical integration of the equations for the magnetic field lines}

The Hamiltonian dynamics associated to the equations (2)-(3) has been integrated using a symplectic integrator with toroidal angle 'time'-step $2 \pi / 3200$.

Figures 5 and 6 represent the radial-type component, $\psi$, of different magnetic field lines as a function of the toroidal angle $\phi$ for the microturbulence model for two values of the relative magnetic perturbation, namely $\varepsilon=$ $2 \cdot 10^{-5}$ and $\varepsilon=10^{-4}$. The non-integrable character of the magnetic field lines is quite manifest from the figures.

However, whereas the behavior of the magnetic field lines looks stochastic for $\varepsilon=10^{-4}$, this is much weaker for $\varepsilon=2 \cdot 10^{-5}$ where the magnetic field trajectories correlate with the $(m, n)$ resonances. As the magnetic braiding gets smaller, the stochasticity in the $\psi$-domain may become locally quite low so that the resonances are not fully disaggregated behaving as cantori. As apparent on the left plot of Figure 5, magnetic field lines can be reflected by remnants or almost intact resonances, possibly of a high order, which induces some memory effect.

In Figure 4, the relative minimal values of the two-wave overlap Chirikov parameter divided by $\varepsilon^{1 / 2}$ are close to 124 about the $(4,1)$ resonance and to 133 about the $(3,1)$ resonance. Being obtained for $\varepsilon=2 \cdot 10^{-5}$, Figure 5 corresponds then to a situation where the Chirikov parameter about the resonances associated to the integer values of $q$, where the chaos is minimal, is about 0.6 , so noticeably below 1. One could therefore be surprised to observe some (at least partial) breaking of the KAM tori about the integer surfaces.

However, it must be emphasized that the classical criterion for chaos - that the Chirikov overlap parameter $s_{1,2}$ between two resonances waves be larger than one - is a crude one and many refinements have been proposed ${ }^{29}$. In particular, even if we had a Hamiltonian system with just two waves, taking into account the secondary resonances arising from the nonlinear beating between the two primary resonances would reduce the effective distance between resonances ${ }^{30}$. A rule of thumb is that connected chaos occurs when $s_{1,2} \simeq 2 / 3$ instead of 1 due to this mechanism. The most sophisticated treatment for the paradigmatic case of two primary resonances consists in taking into account the creation of infinitely many secondary resonances and using renormalization theory to compute the chaos threshold ${ }^{31,32}$. Since this effect of secondary resonances couples here to the existence of a spectrum of primary modes, it is not surprising to observe the onset of chaos for values of the Chirikov overlap parameter below $2 / 3$. Moreover there is some source for additional chaos since the resonances are not purely pendulum sinusoidal waves. This is apparent in the phase space portrait of a microtearing mode that has two close $\mathrm{x}$-points instead of one in the pendulum sinusoidal case (see Figure 3).

The situation of Figure 5 with $\varepsilon=2 \cdot 10^{-5}$ is certainly above but very close to the threshold for connected chaos since one can observe that two magnetic field lines remain contained between the $(3,1)$ and $(4,1)$ resonance radial locations whereas the other two magnetic field lines happen to cross these. For low enough values of $\varepsilon$, the Chirikov parameter between the magnetic resonances eventually becomes small enough in macroscopic domains of the $\psi$-space impeding (normal) diffusion.

\section{MAGNETIC CONFINEMENT TIMES}

\section{A. The magnetic microturbulence picture}

In Sect. II D, from the integration of the magnetic field line trajectories at experimentally relevant values of the relative magnetic perturbation parameter $\varepsilon$ emerges the picture of the microturbulent magnetic transport. Some locally stochastic domains of the phase space are separated by partial transport barriers (cantori) limiting the large scale magnetic diffusion. This suggests that characterizing the transport through a magnetic diffusion coefficient, $D_{\mathrm{m}}$, may not be a universally appropriate approach. Indeed, the presence of cantori should induce a strong locality of the diffusion coefficient: namely $D_{\mathrm{m}}$ depends on the action $\psi$, with locally almost vanishing minima about the $\psi_{m n}$ associated to the cantori. Moreover, the diffusive nature of the transport is unclear. Computing a diffusion coefficient implicitly means that the diffusion is normal, neither sub- nor super-diffusive. An alternative physical indicator, that is a natural indicator in the fusion context where Lawson's criterion is central, would rather be given by the characteristic time spent by the magnetic field lines to visit the phase space, that may be viewed as a confinement time. The benefit of this indicator is that it remains valid even in the absence of (normal) diffusion.

\section{B. First exit times}

A physically meaningful indicator of the magnetic confinement for fusion applications is the residence time of the magnetic field lines inside some inner volume of the device. Indeed, in magnetic confinement devices, electrons are strongly magnetized, so that a breakdown of the magnetic confinement may result in the termination of the electron confinement, or in other words, in a disruption.

To probe the magnetic transport properties within the microturbulence framework, extensive numerical simulations have been performed. Starting from magnetic field lines in the core of the tokamak, more precisely such that $\psi(\phi=0)$ be in the range $[0.05 ; 0.1]$, the number of toroidal turns $\phi_{\text {exit }} /(2 \pi)$ after which $\psi\left(\phi_{\text {exit }}\right)$ becomes larger than 0.9 was computed for a large number of field lines and at different values of the stochasticity parameter $\varepsilon$. Figure 7 represents the distribution of the first exit times $\phi_{\text {exit }}$ in units of toroidal turns for two values of $\varepsilon$. 

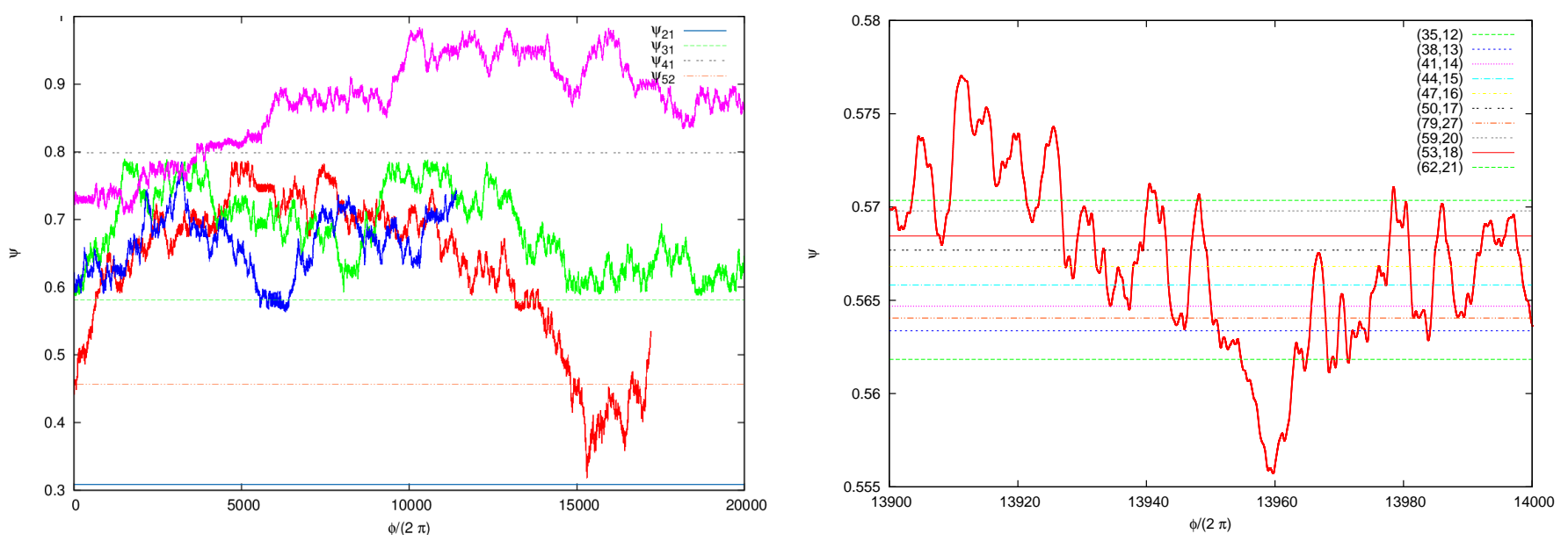

FIG. 5. (Left) Four magnetic field line traces $\psi(\phi)$ for the magnetic microturbulence model with $\varepsilon=2 \cdot 10^{-5}$. (Right) Focus on one of the magnetic field line traces $\psi(\phi)$ being transiently exploring a narrow $\psi$-domain below $\psi_{31} \simeq 0.5816$. The locations in the $\psi$-space of some secondary resonances are indicated.

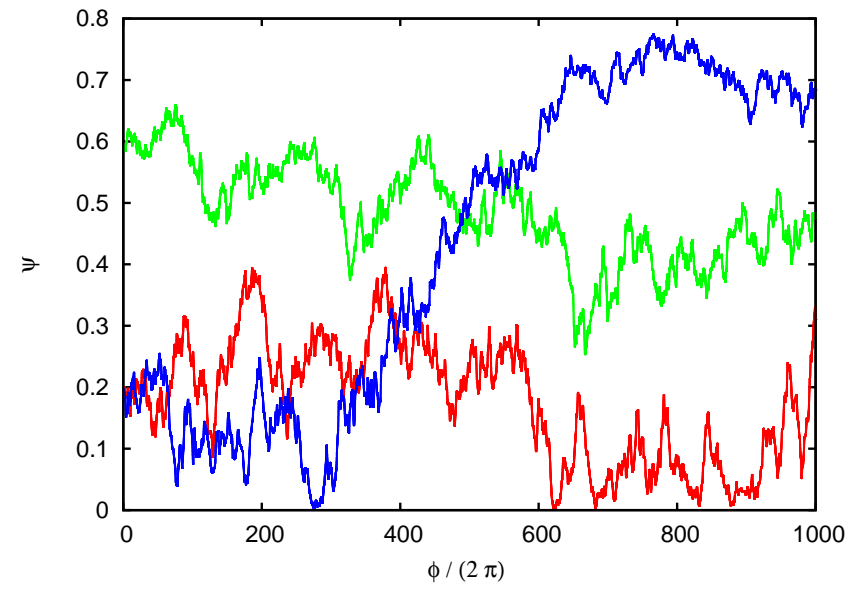

FIG. 6. Three magnetic field line traces $\psi(\phi)$ for the magnetic microturbulence model with $\varepsilon=10^{-4}$.

In Figure 8 is plotted for some values of $\varepsilon$ above $10^{-4}$ the mean exit times computed by launching as many as some tens of thousand initial conditions for the magnetic field lines in the tokamak core. Figure 7 validates the magnetic microturbulence picture depicted in Sect. III A. For values of $\varepsilon$ roughly below $10^{-4}$, the values of the exit times become very large, in the sense that it becomes numerically too demanding to compute them. Moreover as $\varepsilon$ decreases, there is an increasingly growing fraction of the trajectories that never attain the tokamak border, getting glued about low order resonances. As $\varepsilon$ increases, the local Chirikov parameter eventually raises above one in the whole $\psi$ domain and the conditions for the usual diffusion in $\psi$ space are fully satisfied. Indeed, for the range of $\varepsilon$ values considered in Fig. 8, the mean exit times scale with $\varepsilon$ as $\varepsilon^{-2}$. This is the scaling expected from a quasilinear diffusion approach ${ }^{33}$, since $D_{\mathrm{QL}} \propto(\delta B / B)^{2}$ and $\delta B / B \propto \varepsilon$.
The Brownian nature of the behaviour of magnetic field lines may be assessed by comparing the numerically obtained distribution of first exit times with its prediction in the case of Brownian motion. The first passage time distribution of Brownian motion with positive drift takes the form

$$
f(\tau ; \mu, \lambda)=\left(\frac{\lambda}{2 \pi \tau^{3}}\right)^{1 / 2} \exp \left[-\frac{\lambda}{2 \mu^{2} \tau}(\tau-\mu)^{2}\right] .
$$

It can be seen on Figure 9 that the distribution of the numerical exit times in the case $\epsilon=10^{-3}$ is fairly well approximated by a function of the form (14). The next Section will be devoted to some consequences for tokamak physics of the present results.

\section{INDICATIONS FOR A DISRUPTION LIMIT DUE TO MAGNETIC MICROTURBULENCE}

\section{A. Preliminary justification for a static frame}

The magnetic model presented in Section II may be made time-dependent to account for the time variations of field perturbations in real plasmas. Typical fluctuation frequencies have been reported to be in the range $10^{4}$ to $10^{5} \mathrm{~Hz}$. In this Section, our interest will lie however on disruptive-type phenomena taking place on the time scale of tens to hundreds of electron toroidal excursions. For such a short time scale, it is possible to neglect the diffusion effects induced by phase and restrict to a static approach.

Moreover, it is a well-known experimental fact that phase-locking between the rotating modes is one 'favorable' ingredient for disruptions ${ }^{34-36}$. Considering that modes rotate in phase is equivalent in the frame of the modes to consider constant phases. One just proceeds to a Galilean transform to the wave frame. Disruptions 

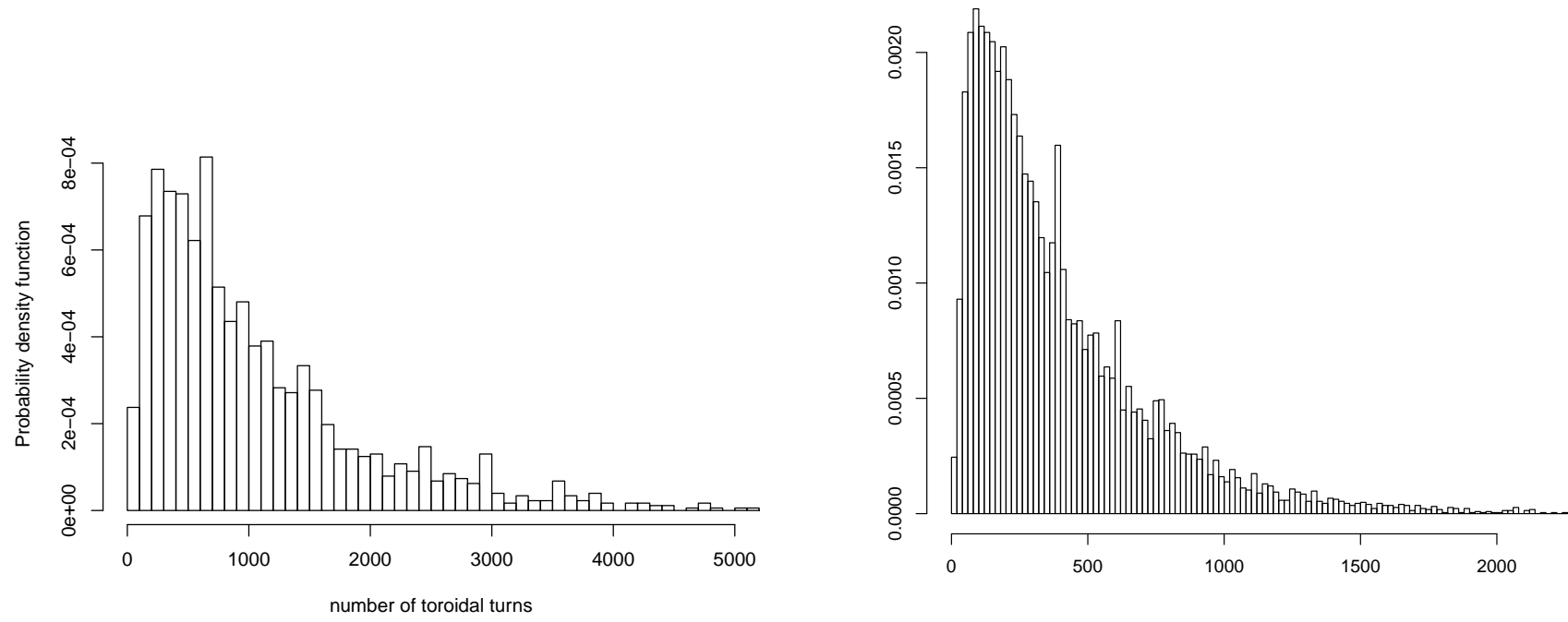

FIG. 7. Probability density function (PDF) of the first exit times for two different values of the stochasticity parameter $\varepsilon$ in the magnetic microturbulence model: (left) for $\varepsilon=3.10^{-4}$, (right) for $\varepsilon=5.10^{-4}$.

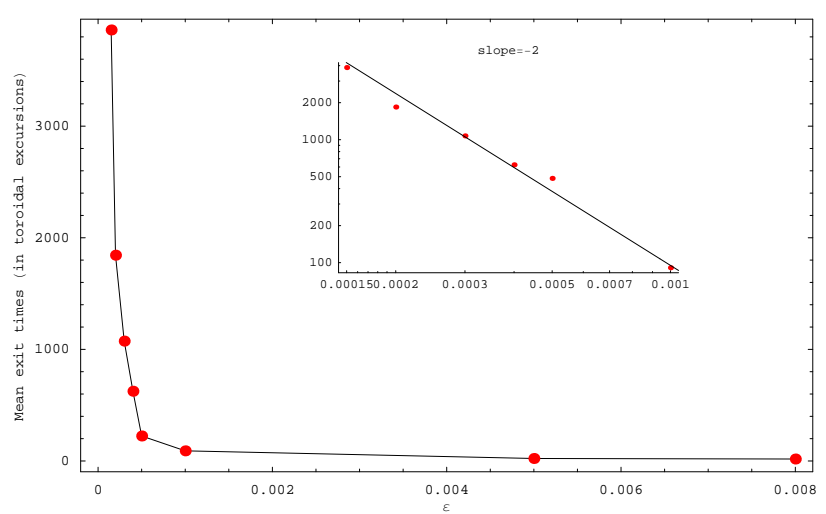

FIG. 8. Mean exit times in number of toroidal turns as a function of $\varepsilon$. The inset shows a log-log representation of the same points together with a line of slope -2 .

have also been observed to take place with slowly rotating modes. Then, with regard to the brevity of the disruptions, treating the disruption scenario in a static frame can be a good approximation.

In any case, the static situation should be the more deleterious, limit scenario for the loss of confinement since one can easily imagine that some differential poloidal rotation in the modes contrarily makes the field lines spend more time moving in the poloidal direction which increases their mean exit times.

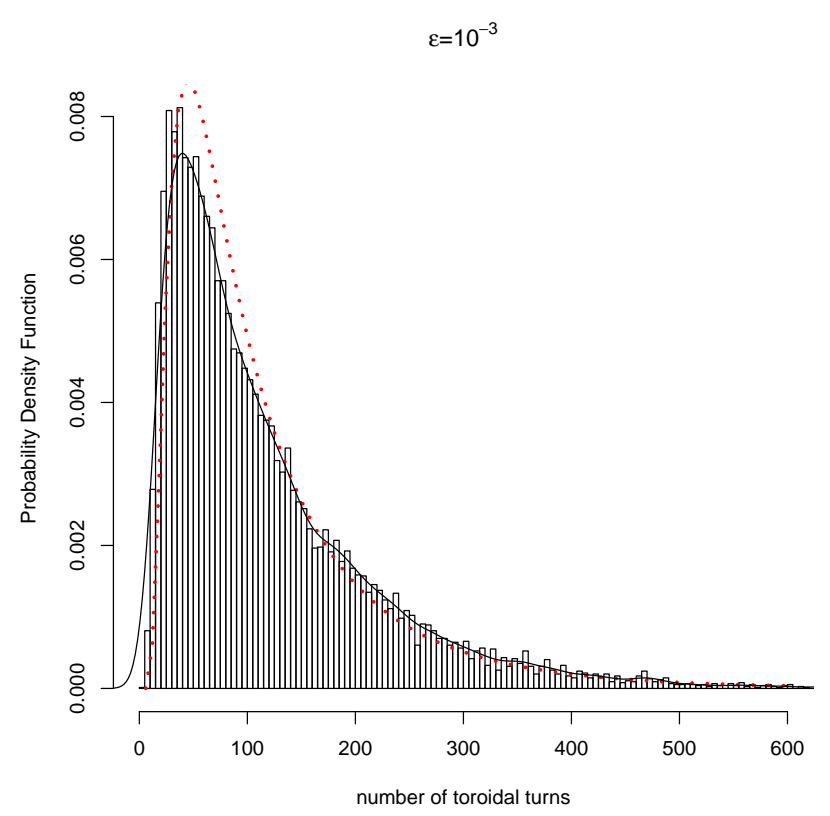

FIG. 9. Probability density function of the first exit times for $\varepsilon=10^{-3}$ in the magnetic microturbulence model (histogram and plain line continuous representation). In dotted line is superimposed the first passage time distribution of Brownian motion with positive drift given in Eq. (14) having the same mean and variance as the data. 


\section{B. Magnetic turbulence-driven disruption limit}

In tokamak physics, there exist some dramatically swift phenomena taking place on the lapse of time of some tens to a couple of hundreds of microseconds. These are the collapse phases of sawteeth, edge localized modes and disruptions. All these phenomena may be interpreted as transient, benign or severe losses of the magnetic confinement and are mediated by the lighter charged particles of the plasma, namely by the electrons. In a tokamak such as JET, the electron thermal velocity at a temperature of $10 \mathrm{keV}$ is about $4.10^{7} \mathrm{~m} . \mathrm{s}^{-1}$ so that the characteristic time spent by electrons to make a toroidal excursion is $0.5 \mu \mathrm{s}$. Consequently, a lapse of time of $100 \mu \mathrm{s}$ amounts to 200 electron toroidal excursions. It is quite instructive to see on Figure 8 that 200 toroidal excursions is the order of magnitude of the qualitative threshold mean exit time separating a quick loss of the magnetic confinement for $\varepsilon>\varepsilon_{\text {th }}$ from a much better magnetic confinement provided the perturbation parameter $\varepsilon$ becomes some fraction of $\varepsilon_{\mathrm{th}}$. From the Figure, one may retain the value $5.10^{-4}$ for $\varepsilon_{\mathrm{th}}$. The magnetic perturbation $\delta B / B$ is of order $\varepsilon$ with the spectrum of modes used here. Its value associated to $\varepsilon=5 \cdot 10^{-4}$ could be derived using Eq. (13).

Looking at Figure 8 comes the impression of a threshold effect in the behaviour of the mean exit time as a function of $\varepsilon$. We shall here examine its origin.

In the regime where the resonance overlap is sufficient to ensure the complete stochasticity of the magnetic field lines and the validity of the quasilinear approximation, the mean exit 'times' $\left\langle\phi_{\text {exit }}\right\rangle$ scales as $\varepsilon^{-2}$ : there exists some constant $C>0$ such that $\left\langle\phi_{\text {exit }}\right\rangle=C^{2} \varepsilon^{-2}$. Converting the toroidal angle to a real time $\tau$ through the use of the electron parallel velocity as we have just done and using the fact that $\delta B / B$ is proportional to $\varepsilon$, this means that the characteristic exit time scales with respect to the relative magnetic perturbation as $\left\langle\tau_{\text {exit }}\right\rangle \propto(\delta B / B)^{-2}$.

Consequently, all other things being equal, a small change in the magnetic perturbation $\Delta(\delta B / B)$ is associated to a much larger change in the mean exit time $\Delta\left\langle\tau_{\text {exit }}\right\rangle$ since

$$
\frac{\Delta\left\langle\tau_{\text {exit }}\right\rangle}{\left\langle\tau_{\text {exit }}\right\rangle}=-2\left(\frac{\delta B}{B}\right)^{-1} \Delta\left(\frac{\delta B}{B}\right)
$$

where $\delta B / B$ is a (very) small parameter. From this it follows that a relatively small change in the magnetic perturbation is associated to a much larger relative variation in the mean confinement time. This sensitivity effect is beneficial when the magnitude of the magnetic perturbation is reduced since the confinement time may drastically increase but this effect is deleterious when the magnetic perturbation is increased, by intrinsic or extrinsic routes, as the confinement time may drop to the point where a macroscopic (major) disruption occurs and confinement is lost.

\section{DISCUSSION}

In the present study, the magnetic perturbation $\delta B / B$, or equivalently the parameter $\varepsilon$, is a control parameter which may take arbitrary values. In the framework of magnetic microturbulence, magnetic field lines become stochastic in a macroscopic fraction of the tokamak volume when the Chirikov parameter computed for any two resonant waves $(m, n)$ and $\left(m^{\prime}, n^{\prime}\right)$ is roughly above $2 / 3^{29}$. This has been shown to take place for the values of $\delta B / B$ that have been experimentally measured by cross polarization scattering in Tore Supra ${ }^{1}$ or by using the poloidal sweep of a heavy ion probing beam recently in JIPPT-IIU ${ }^{2}$. These tokamaks therefore operate in regimes where the stochastic hypothesis necessary to derive a Fokker-Planck diffusive approach, like the quasilinear theory, is satisfied. Consistently, both the Tore Supra and JIPPT-IIU teams have verified that the experimentally measured levels of magnetic perturbation were compatible with their experimentally measured electron heat diffusivities $\chi_{e}$ when using the quasilinear expression for $\chi_{e}{ }^{33}$.

Predicted here is the existence of some qualitative threshold on $\delta B / B$ in this stochastic regime above which the mean confinement exit times become too low to be sustainable by the tokamak plasma. The sensitivity in $\delta B / B$ is reflected by Eq. (15). One may wonder whether this disruptive-like limit may actually be reached. Indeed, once there is an overall stochasticity of magnetic field lines, there is some self-healing process coming into play in the sense that the stochasticity of magnetic field lines quenches the thermal gradients that are a source of instability for the microtearing modes and may regulate the magnetic microturbulence. However, collisionality may remain as a source of destabilization of microtearing modes.

These results should be put in perspective with the recent achievements on runaway electron suppression above some magnetic turbulence threshold reported by Zeng et $a l .37$. These experimental measurements done on TEXTOR have shown that magnetic turbulence is mainly contributed from the background plasma and that its level does strongly dependent on the toroidal magnetic field and plasma density. Combining the two scaling laws obtained by the authors comes the experimental scaling for the level of magnetic turbulence

$$
\frac{\delta B}{B} \propto B^{-3} \sqrt{n_{e}}
$$

where $n_{e}$ stands for the line averaged central density. It follows from Eq. (16) that the previous thresholdlike limit on magnetic turbulence for disruption should also be a density limit and that a high magnetic field is beneficial. 


\section{ACKNOWLEDGMENTS}

Preliminary discussions with S.S. Abdullaev and D. Constantinescu and assistance on parallel computing by A.F. Lifschitz are gratefully acknowledged. This work was carried out within the framework the French Research Federation for Fusion Studies.

${ }^{1}$ X. L. Zou, L. Colas, M. Paume, J. M. Chareau, L. Laurent, P. Devynck, and D. Gresillon Phys. Rev. Lett. 75, 1090 (1995).

${ }^{2}$ Y. Hamada, T. Watari, A. Nishizawa, O. Yamagishi, K. Narihara, K. Ida, Y. Kawasumi, T. Ido, M. Kojima, K. Toi and the JIPPTIIU Group, Nucl. Fusion 55, 043008 (2015).

${ }^{3}$ W. M. Nevins, E. Wang, and J. Candy, Phys. Rev. Lett. 106, 065003 (2011).

${ }^{4}$ A.J. Lichtenberg, Nucl. Fus. 24, 1277 (1984).

${ }^{5}$ V. Igochine, O. Dumbrajs, H. Zohm, and ASDEX Upgrade Team, Nucl. Fusion 48, 062001 (2008).

${ }^{6}$ M.-C. Firpo, W. Ettoumi, R. Farengo, H. Ferrari, P.L. GarcíaMartínez, and A.F. Lifschitz, Phys. Plasmas 20, 072305 (2013).

${ }^{7}$ W. Ettoumi, Ph-D thesis, Ecole Polytechnique (2013).

${ }^{8}$ H. Doerk, F. Jenko, M. J. Pueschel, and D. R. Hatch, Phys. Rev. Lett. 106, 155003 (2011).

${ }^{9}$ W. Guttenfelder, J. Candy, S. M. Kaye, W. M. Nevins, E. Wang, R. E. Bell, G. W. Hammett, B. P. LeBlanc, D. R. Mikkelsen, and H. Yuh, Phys. Rev. Lett. 106, 155004 (2011).

${ }^{10}$ D. R. Hatch, M. J. Pueschel, F. Jenko, W. M. Nevins, P. W. Terry, and H. Doerk, Phys. Rev. Lett. 108, 235002 (2012).

${ }^{11} \mathrm{P}$ Manz, J E Boom, E Wolfrum, G Birkenmeier, I G J Classen, N C Luhmann Jr, U Stroth and the ASDEX Upgrade Team, Plasma Phys. Control. Fusion 56, 035010 (2014).

${ }^{12}$ B. Cambon, X. Leoncini, M. Vittot, R. Dumont and X. Garbet, Chaos 24, 033101 (2014).

${ }^{13}$ J.R. Cary and R.G. Littlejohn, Annals of Physics 151, 1-34 (1983).

${ }^{14}$ B.V.Chirikov, Phys. Rep. 52, 263 (1979).

${ }^{15}$ W.D. D'haeseleer, W.N.G. Hitchon, J.D. Callen and J.L. Shohet, Flux coordinates and magnetic field structure (Springer, Berlin, 1991).

${ }^{16}$ A. H. Boozer, Phys. Fluids 26, 1288-1291 (1983).

${ }^{17}$ P. J. Morrison, Phys. Plasmas 7, 2279 (2000).

${ }^{18}$ M.-C. Firpo and D. Constantinescu, Phys. Plasmas 18, 032506 (2011).
${ }^{19}$ T. E. Evans, R. A. Moyer, K. H. Burrell, M. E. Fenstermacher, I. Joseph, A. W. Leonard, T. H. Osborne, G. D. Porter, M. J. Schaffer, P. B. Snyder, P. R. Thomas, J. G. Watkins, and W. P. West, Nat. Phys. 2, 419 (2006).

${ }^{20}$ S. S. Abdullaev, Construction of Mappings for Hamiltonian Systems and Their Applications (Springer, 2006).

${ }^{21}$ K.-H. Spatschek, High Temperature Plasmas: Theory and Mathematical Tools for Laser and Fusion Plasmas (Wiley, Weinheim, 2012).

${ }^{22}$ H. Wobig, Z. Naturforsch. 42a, 1054 (1987).

${ }^{23}$ A. Punjabi, A. Verma, and A. Boozer, Phys. Rev. Lett. 69, 3322 (1992)

${ }^{24}$ S. S. Abdullaev and G. M. Zaslavsky, Phys. Plasmas 2, 4533 (1995).

${ }^{25}$ S. S. Abdullaev, K. H. Finken, A. Kaleck, and K. H. Spatschek, Phys. Plasmas 5, 196-210 (1998).

${ }^{26}$ R. Balescu, M. Vlad, and F. Spineanu, Phys. Rev. E 58, 951 964 (1998).

${ }^{27}$ S.S. Abdullaev, Nucl. Fusion 50, 034001 (2010).

${ }^{28}$ T. H. Stix, Phys. Rev. Lett. 30, 833 (1973).

${ }^{29}$ J. D. Meiss, Differential Dynamical Systems, SIAM Philadelphia (2007).

${ }^{30}$ A. J. Lichtenberg and M. A. Lieberman, Regular and Chaotic Motion, New York, Springer-Verlag (1992).

${ }^{31}$ D. F. Escande, Phys. Rep. 121, 165-261 (1985).

${ }^{32}$ R. S. MacKay, Renormalization in area-preserving maps, Singapore, World Scientific (1993).

${ }^{33}$ A. B. Rechester and M. N. Rosenbluth, Phys. Rev. Lett. 40, 38 (1978).

${ }^{34}$ J.A. Snipes, D.J. Campbell, P.S. Haynes, T.C. Hender, M. Hugon, P.J. Lomas, N.J. Lopes Cardozo, M.F.F. Nave and F.C. Schüller, Nucl. Fusion 28, 1085 (1988).

${ }^{35}$ P.C. de Vries, M.F. Johnson, B. Alper, P. Buratti, T.C. Hender, H.R. Koslowski, V. Riccardo and JET-EFDA Contributors, Nuclear Fusion 51, 053018 (2011).

${ }^{36}$ F. A. Volpe, A. Hyatt, R. J. La Haye, M. J. Lanctot, J. Lohr, R. Prater, E. J. Strait, and A. Welander, to appear in Phys. Rev. Lett. (2015).

${ }^{37}$ L. Zeng, H. R. Koslowski, Y. Liang, A. Lvovskiy, M. Lehnen, D. Nicolai, J. Pearson, M. Rack, H. Jaegers, K. H. Finken, K. Wongrach, Y. Xu, and the TEXTOR team, Phys. Rev. Lett. 110, 235003 (2013). 